Research Article

\title{
Convolution Properties of a Subclass of Analytic Univalent Functions
}

\author{
Saurabh Porwal \\ Department of Mathematics, UIET, CSJM University, Kanpur 208024, India \\ Correspondence should be addressed to Saurabh Porwal; saurabhjcb@rediffmail.com \\ Received 26 September 2013; Accepted 19 November 2013; Published 2 February 2014 \\ Academic Editors: K. Lurie and J.-L. Wu \\ Copyright (C) 2014 Saurabh Porwal. This is an open access article distributed under the Creative Commons Attribution License, \\ which permits unrestricted use, distribution, and reproduction in any medium, provided the original work is properly cited. \\ The main objective of the present paper is to investigate some interesting properties on convolution and generalized convolution \\ of functions for the classes $R(n, \alpha)$ and $\bar{R}(n, \alpha)$. Our results improve the results of previous authors.
}

\section{Introduction}

Let $A$ denote the class of functions $f$ of the form

$$
f(z)=z+\sum_{k=2}^{\infty} a_{k} z^{k}
$$

which are analytic in the open unit disk $U=\{z: z \in C$ and $|z|<1\}$ and satisfy the normalization condition $f(0)=$ $f^{\prime}(0)-1=0$. Let $S$ be the subclass of $A$ consisting of functions of the form (1) which are also univalent in $U$. Further, $T$ denote the subclass of $A$ consisting of functions $f(z)$ of the form

$$
f(z)=z-\sum_{k=2}^{\infty}\left|a_{k}\right| z^{k}
$$

Now for $0 \leq \alpha<1, n \in N=\{1,2,3, \ldots\}$, and $z \in U$, suppose that $R(n, \alpha)$ denotes the family of analytic univalent functions $f$ of the form (1) such that

$$
\operatorname{Re}\left\{\frac{D^{n} f(z)}{z}\right\}>\alpha,
$$

where $D^{n}$ stands for the Salagean operator introduced by Salagean in [1].

Further, let the subclass $\bar{R}(n, \alpha)$ consist of functions $f$ in $R(n, \alpha)$ such that $f(z)$ is of the form (2).
Clearly, if $0 \leq \alpha_{1} \leq \alpha_{2}<1$, then

$$
R\left(n, \alpha_{2}\right) \subseteq R\left(n, \alpha_{1}\right),
$$

and for $m, n \in N$, such that $m \geq n$, then

$$
R(m, \alpha) \subseteq R(n, \alpha) .
$$

The Hadamard product of two functions $f(z)$ of the form (1) and $g(z)$ is of the form

$$
g(z)=z+\sum_{k=2}^{\infty} b_{k} z^{k}
$$

as

$$
(f * g)(z)=f(z) * g(z)=z+\sum_{k=2}^{\infty} a_{k} b_{k} z^{k},
$$

and for the modified Hadamard product (quasi-convolution) of two functions $f(z)$ of the form (2) and

$$
g(z)=z-\sum_{k=2}^{\infty}\left|b_{k}\right| z^{k}
$$

we define their convolution as

$$
(f * g)(z)=f(z) * g(z)=z-\sum_{k=2}^{\infty}\left|a_{k} b_{k}\right| z^{k} .
$$

In the present paper, we obtain a number of results on convolution and generalized convolution for the classes $R(n, \alpha)$ and $\bar{R}(n, \alpha)$. It is worthy to note that our results are quite new and not explored in the literature. 


\section{Main Results}

We first mention a sufficient condition for the function $f$ of the form (1) belonging to the class $R(n, \alpha)$ given by the following result which can be established easily.

Theorem 1. Let the function $f(z)$ be given by (1). Furthermore, let

$$
\sum_{k=2}^{\infty} k^{n}\left|a_{k}\right| \leq 1-\alpha
$$

where $0 \leq \alpha<1$ and $n \in N$. Then $f \in R(n, \alpha)$.

In the following theorem, it is proved that the condition (10) is also necessary for functions $f(z)$ of the form (2).

Theorem 2. Let $f(z)$ be given by (2). Then $f \in \bar{R}(n, \alpha)$, if and only if

$$
\sum_{k=2}^{\infty} \frac{k^{n}}{1-\alpha}\left|a_{k}\right| \leq 1,
$$

where $0 \leq \alpha<1$ and $n \in N$.

Proof. The if part follows from Theorem 1, so we only need to prove the "only if" part of the theorem. To this end, for functions $f$ of the form (2), we notice that the condition

$$
\operatorname{Re}\left\{\frac{D^{n} f(z)}{z}\right\}>\alpha
$$

is equivalent to

$$
\operatorname{Re}\left\{1-\sum_{k=2}^{\infty} k^{n}\left|a_{k}\right| z^{k-1}\right\}>\alpha .
$$

The above required condition must hold for all values of $z$ in $U$. Upon choosing the values of $z$ on the positive real axis and making $z \rightarrow 1^{-}$, we must have

$$
1-\sum_{k=2}^{\infty} k^{n}\left|a_{k}\right| \geq \alpha
$$

which is the required condition.

Several authors such as [2-6] studied the convolution properties for the functions with negative as well as positive coefficients only. Their results do not say anything for the function of the form (1). It is therefore natural to ask whether their results can be improved for function of the form (1). In our next theorem, we establish a result on convolution which improves the results of previous authors [2-6] to the case when $f$ is of the form (1). It is worth mentioning that the technique employed by us is entirely different from the previous authors. For this, we will require the following definition and lemmas.

Definition 3. A sequence $\left\{c_{k}\right\}_{0}^{\infty}$ of nonnegative numbers is said to be a convex null sequence if $c_{k} \rightarrow 0$ as $k \rightarrow \infty$ and

$$
c_{0}-c_{1} \geq c_{1}-c_{2} \geq \cdots \geq c_{k}-c_{k+1} \geq \cdots \geq 0 .
$$

Lemma 4. Let $\left\{c_{k}\right\}_{0}^{\infty}$ be a convex null sequence. Then the function

$$
q_{1}(z)=\frac{c_{0}}{2}+\sum_{k=1}^{\infty} c_{k} z^{k}
$$

is analytic in $U$ and $\operatorname{Re} q_{1}(z)>0, z \in U$.

Lemma 5. Let $P(z)$ be analytic in $U, P(0)=1$, and $\operatorname{Re}\{P(z)\}>$ $(1 / 2)$ in $U$. For functions $F$ analytic in $U$, the convolution function $P * F$ takes values in the convex hull of the image on $U$ under $F$.

Lemma 4 is due to Fejér [7]. The assertion of Lemma 5 readily follows by using the Herglotz representation for $P(z)$.

Lemma 6. For $0 \leq \alpha<1, n \in N-\{1\}$, let $f(z) \in R(n, \alpha)$. Then

$$
\operatorname{Re}\left\{\frac{f(z)}{z}\right\}>\frac{1}{2}, \quad z \in U .
$$

Proof. Let $f(z)$ be given by (1). Since $f(z) \in R(n, \alpha)$, hence, by definition

$$
\operatorname{Re}\left\{\frac{D^{n} f(z)}{z}\right\}>\alpha, \quad z \in U,
$$

which is equivalent to

$$
\operatorname{Re}\left\{1-\alpha+\sum_{k=2}^{\infty} k^{n} a_{k} z^{k-1}\right\}>0
$$

and hence,

$$
\operatorname{Re}\left\{1+\frac{1}{2} \sum_{k=2}^{\infty} \frac{k^{n}}{1-\alpha} a_{k} z^{k-1}\right\}>\frac{1}{2} .
$$

We observe that the sequence $\left\{c_{k}\right\}_{0}^{\infty}$ defined by $c_{0}=1$ and $c_{k}=2(1-\alpha) /(k+1)^{n}, k \geq 1, n \geq 2$, is a convex null sequence; we have in view of Lemma 4 that

$$
\operatorname{Re}\left\{1+2 \sum_{k=2}^{\infty} \frac{1-\alpha}{k^{n}} a_{k} z^{k-1}\right\}>\frac{1}{2} \text {. }
$$

Now

$$
\frac{f(z)}{z}=\left[1+\frac{1}{2} \sum_{k=2}^{\infty} \frac{k^{n}}{1-\alpha} a_{k} z^{k-1}\right] *\left[1+2 \sum_{k=2}^{\infty} \frac{1-\alpha}{k^{n}} z^{k-1}\right]
$$

and making use of (20), (21), and Lemma 5, we conclude that

$$
\operatorname{Re}\left\{\frac{f(z)}{z}\right\}>\frac{1}{2} \text {. }
$$

Theorem 7. If $f(z)=z+\sum_{k=2}^{\infty} a_{k} z^{k} \in R(n, \alpha)$ and $g(z)=$ $z+\sum_{k=2}^{\infty} b_{k} z^{k} \in R(m, \beta)$, where $0 \leq \beta \leq \alpha<1, n \in N, m \in$ $N-\{1\}$, then so does their Hadamard product (convolution)

$$
h(z)=(f * g)(z)=z+\sum_{k=2}^{\infty} a_{k} b_{k} z^{k} \in R(n, \alpha) \text {. }
$$


Proof. To prove that $h(z) \in R(n, \alpha)$ we have to show that

$$
\operatorname{Re}\left\{\frac{D^{n} h(z)}{z}\right\}>\alpha,
$$

which is equivalent to

$$
\operatorname{Re}\left\{1-\alpha+\sum_{k=2}^{\infty} k^{n} a_{k} b_{k} z^{k-1}\right\}>0
$$

or

$$
\operatorname{Re}\left\{1+\frac{1}{2} \sum_{k=2}^{\infty} \frac{k^{n}}{1-\alpha} a_{k} b_{k} z^{k-1}\right\}>\frac{1}{2} .
$$

Since $f \in R(n, \alpha)$ from (20) we have

$$
\operatorname{Re}\left\{1+\frac{1}{2} \sum_{k=2}^{\infty} \frac{k^{n}}{1-\alpha} a_{k} z^{k-1}\right\}>\frac{1}{2}
$$

and since $g \in R(m, \beta)$, from Lemma 6 , we have

$$
\operatorname{Re}\left\{\frac{g(z)}{z}\right\}>\frac{1}{2}
$$

or

$$
\operatorname{Re}\left\{1+\sum_{k=2}^{\infty} b_{k} z^{k-1}\right\}>\frac{1}{2}
$$

From (28), (30), and Lemma 5 we immediately have (27).

This establishes the proof of Theorem 7.

In our next result we improve the results of Theorem 7 for functions of the form (2).

Theorem 8. Let the functions $f(z), g(z)$ defined by (2), (8) be in the classes $\bar{R}(n, \alpha), \bar{R}(m, \beta)$, respectively, where $m, n \in N$, $0 \leq \beta<1$, and $0 \leq \alpha<1$; then $f * g$ defined by (9) is in the class $\bar{R}(m+n, \eta)$, where $\eta=\alpha+\beta-\alpha \beta$.

Proof. Since $f(z) \in \bar{R}(n, \alpha)$, then, by Theorem 2, we have

$$
\sum_{k=2}^{\infty} \frac{k^{n}}{1-\alpha}\left|a_{k}\right| \leq 1
$$

Similarly, since $g(z) \in \bar{R}(m, \beta)$, we have

$$
\sum_{k=2}^{\infty} \frac{k^{m}}{1-\beta}\left|b_{k}\right| \leq 1
$$

Therefore, from (31), we have

$$
\frac{k^{n}}{1-\alpha}\left|a_{k}\right| \leq 1, \quad k=2,3, \ldots
$$

Now, for the convolution function $f * g$ we have

$$
\begin{aligned}
\sum_{k=2}^{\infty} \frac{k^{m+n}}{1-\eta}\left|a_{k} b_{k}\right| & \leq \sum_{k=2}^{\infty} \frac{k^{m}}{1-\beta}\left|b_{k}\right|, \quad(\operatorname{using}(33)) \\
& \leq 1,(\operatorname{using}(32)) .
\end{aligned}
$$

Thus, the proof of Theorem 8 is established.
Remark 9. From (4) and (5), we see that

$$
\begin{aligned}
& \bar{R}(m+n, \eta) \subseteq \bar{R}(n, \alpha), \\
& \bar{R}(m+n, \eta) \subseteq \bar{R}(m, \beta) .
\end{aligned}
$$

Thus, the result of Theorem 8 provides smaller class in comparison to the class given by Theorem 7 .

Theorem 10. Let the functions $f_{i}(z)$ defined as

$$
f_{i}(z)=z-\sum_{k=2}^{\infty}\left|a_{k, i}\right| z^{k}
$$

belong to the class $\bar{R}\left(n_{i}, \alpha_{i}\right)$ for every $i=1,2, \ldots, q$; then the convolution $f_{1} * f_{2} * \cdots f_{q}$ belongs to the class $\bar{R}\left(\sum_{i=1}^{q} n_{i}, \epsilon\right)$, where $\epsilon=1-\prod_{i=1}^{q}\left(1-\alpha_{i}\right)$.

Proof. The proof of the above theorem is much akin to that of Theorem 8 . Hence, we omit the details involved.

For any real numbers $p$ and $q$, we define that the generalized convolution for functions $f(z)$ and $g(z)$ is of the form (2) and (8) as

$$
(f \Delta g)(p, q ; z)=z-\sum_{k=2}^{\infty}\left|a_{k, 1}\right|^{p}\left|a_{k, 2}\right|^{q} z^{k}
$$

In the special case, if we take $p=q=1$, then we have

$$
(f \Delta g)(1,1 ; z)=(f * g)(z), \quad(z \in U) .
$$

Theorem 11. If the functions $f(z)$ and $g(z)$ defined by (2) and (8) are in the classes $\bar{R}\left(n, \alpha_{1}\right)$ and $\bar{R}\left(n, \alpha_{2}\right)$, respectively, then

$$
(f \Delta g)\left(\frac{1}{p}, \frac{1}{q} ; z\right) \in \bar{R}(n, \alpha)
$$

where $p>1,(1 / p)+(1 / q)=1$ and $\alpha=1-\left(1-\alpha_{1}\right)^{1 / p}(1-$ $\left.\alpha_{2}\right)^{1 / q}$

Proof. Since $f(z) \in \bar{R}\left(n, \alpha_{1}\right)$, by using Theorem 2, we have

$$
\sum_{k=2}^{\infty} \frac{k^{n}}{1-\alpha_{1}}\left|a_{k}\right| \leq 1 .
$$

From (40) we have

$$
\left\{\sum_{k=2}^{\infty} \frac{k^{n}}{1-\alpha_{1}}\left|a_{k}\right|\right\}^{1 / p} \leq 1 .
$$

Similarly for $g \in \bar{R}\left(n, \alpha_{2}\right)$ we have

$$
\left\{\sum_{k=2}^{\infty} \frac{k^{n}}{1-\alpha_{2}}\left|b_{k}\right|\right\}^{1 / q} \leq 1 .
$$


Now

$$
\begin{aligned}
& \sum_{k=2}^{\infty}\left(\frac{k^{n}}{1-\alpha_{1}}\right)^{1 / p}\left(\frac{k^{n}}{1-\alpha_{2}}\right)^{1 / q}\left|a_{k}\right|^{1 / p}\left|b_{k}\right|^{1 / q} \\
& \quad \leq\left\{\sum_{k=2}^{\infty}\left(\frac{k^{n}}{1-\alpha_{1}}\right)\left|a_{k}\right|\right\}^{1 / p}\left\{\sum_{k=2}^{\infty}\left(\frac{k^{n}}{1-\alpha_{2}}\right)\left|b_{k}\right|\right\}^{1 / q}
\end{aligned}
$$$$
\text { (using Hölder s inequality) }
$$

$\leq 1$, (using (41) and (42)).

Since

$$
(f \Delta g)\left(\frac{1}{p}, \frac{1}{q} ; z\right)=z-\sum_{k=2}^{\infty}\left|a_{k}\right|^{1 / p}\left|b_{k}\right|^{1 / q} z^{k},
$$

it suffices to show that $(f \Delta g)((1 / p),(1 / q) ; z) \in \bar{R}(n, \alpha)$ if

$$
\sum_{k=2}^{\infty}\left(\frac{k^{n}}{1-\alpha}\right)\left|a_{k}\right|^{1 / p}\left|b_{k}\right|^{1 / q} \leq 1
$$
by

For this we have to show that L.H.S. of (45) is bounded

$$
\sum_{k=2}^{\infty}\left(\frac{k^{n}}{1-\alpha_{1}}\right)^{1 / p}\left(\frac{k^{n}}{1-\alpha_{2}}\right)^{1 / q}\left|a_{k}\right|^{1 / p}\left|b_{k}\right|^{1 / q}
$$

which is equivalent to $\alpha \leq 1-\left(1-\alpha_{1}\right)^{1 / p}\left(1-\alpha_{2}\right)^{1 / q}$.

In our next result we improve the result of Theorem 11 for the case when $p$ and $q$ are any real numbers such that $p>0$, $q>1$.

Theorem 12. For $0 \leq \alpha_{1} \leq \alpha_{2}<1, p>0, q>1$, let $f(z)$ and $g(z)$ of the form (2) and (8) belong to the classes $\bar{R}\left(n, \alpha_{1}\right)$ and $\bar{R}\left(n, \alpha_{2}\right)$, respectively; then

$$
(f \Delta g)(p, q ; z) \in \bar{R}\left(n, \alpha_{2}\right) \subseteq \bar{R}\left(n, \alpha_{1}\right) .
$$

Proof. Since $f(z) \in \bar{R}\left(n, \alpha_{1}\right)$, by using Theorem 2, we have

$$
\sum_{k=2}^{\infty} \frac{k^{n}}{1-\alpha_{1}}\left|a_{k}\right| \leq 1
$$

or $\left|a_{k}\right| \leq 1, k \geq 2$.

Equivalently, $\left|a_{k}\right|^{p} \leq 1, k \geq 2$, and since $g(z) \in \bar{R}\left(n, \alpha_{2}\right)$, we have

$$
\sum_{k=2}^{\infty} \frac{k^{n}}{1-\alpha_{2}}\left|b_{k}\right| \leq 1
$$

or

$$
\left\{\sum_{k=2}^{\infty} \frac{k^{n}}{1-\alpha_{1}}\left|a_{k}\right|\right\}^{q} \leq 1
$$

Now

$$
\begin{aligned}
& \sum_{k=2}^{\infty}\left(\frac{k^{n}}{1-\alpha_{2}}\right)\left|a_{k}\right|^{p}\left|b_{k}\right|^{q} \\
& \quad \leq \sum_{k=2}^{\infty}\left(\frac{k^{n}}{1-\alpha_{2}}\right)\left|b_{k}\right|^{q} \\
& \leq \sum_{k=2}^{\infty}\left(\frac{k^{n}}{1-\alpha_{2}}\right)^{q}\left|a_{k+p, 2}\right|^{q}, \quad(q>1) \\
& \quad \leq\left\{\sum_{k=2}^{\infty}\left(\frac{k^{n}}{1-\alpha_{2}}\right)\left|b_{k}\right|^{q}\right.
\end{aligned}
$$$$
\leq 1 \text {. }
$$

Therefore, $(f \Delta g)(r, s ; z) \in \bar{R}\left(n, \alpha_{2}\right)$.

Remark 13. Here we give some open problems for the readers.

(1) Find inf $p, q \in R$ and $\sup \alpha \in[0,1)$ such that Theorem 12 holds.

(2) The result of Theorem 12 holds only for functions of the form (2); that is, the coefficients of expansion are negative. Therefore, it is natural to ask what is the analogue results for the function of the form (1).

\section{Conflict of Interests}

The author declares that there is no conflict of interests regarding the publication of this paper.

\section{References}

[1] G. S. Salagean, "Subclasses of univalent functions, complex analysis-fifth romanian finish seminar," Bucharest, vol. 1, pp. 362372,1983

[2] M. Acu, "On some analytic functions with negative coefficients," General Math, vol. 15, no. 2-3, pp. 190-200, 2007.

[3] R. M. Ali, M. H. Khan, V. Ravichandran, and K. G. Subramanian, "A class of multivalent functions with negative coefficients defined by convolution," Bulletin of the Korean Mathematical Society, vol. 43, no. 1, pp. 179-188, 2006.

[4] H. E. Darwish, "The quasi-Hadamard product of certain starlike and convex functions," Applied Mathematics Letters, vol. 20, no. 6, pp. 692-695, 2007.

[5] B. A. Frasin, "Quasi-Hadamard product of certain classes of uniformly analytic functions," General Math, vol. 16, no. 2, pp. 29-35, 2007.

[6] J. Nishiwaki and S. Owa, "Convolutions for certain analytic functions," General Math, vol. 15, no. 2-3, pp. 38-51, 2007.

[7] L. Fejér, "Über die Positivität von Summen, die nach trigonometrischen oder Legendreschen Funktionen fortschreiten," Acta Scientiarum Mathematicarum, vol. 2, pp. 75-86, 1925. 


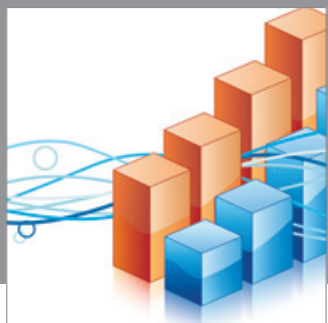

Advances in

Operations Research

mansans

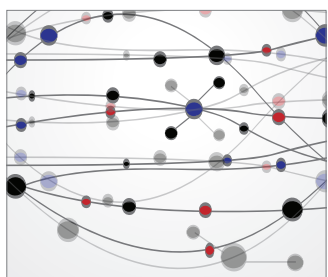

The Scientific World Journal
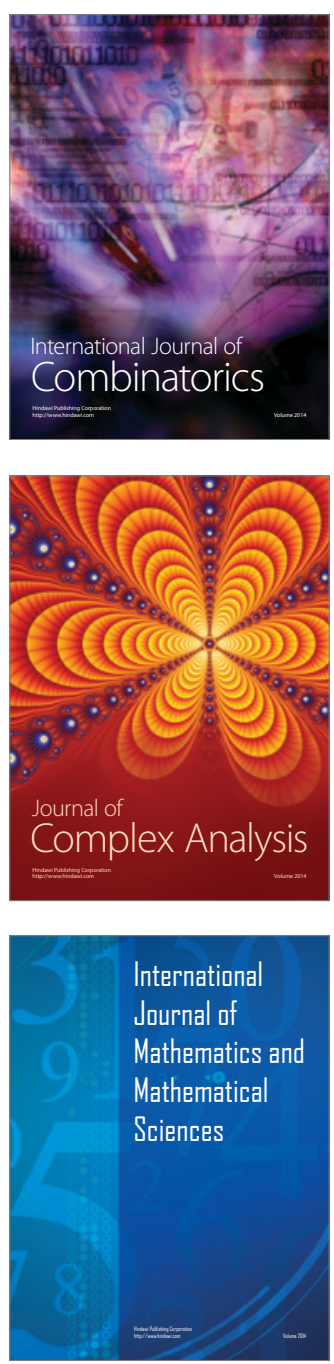
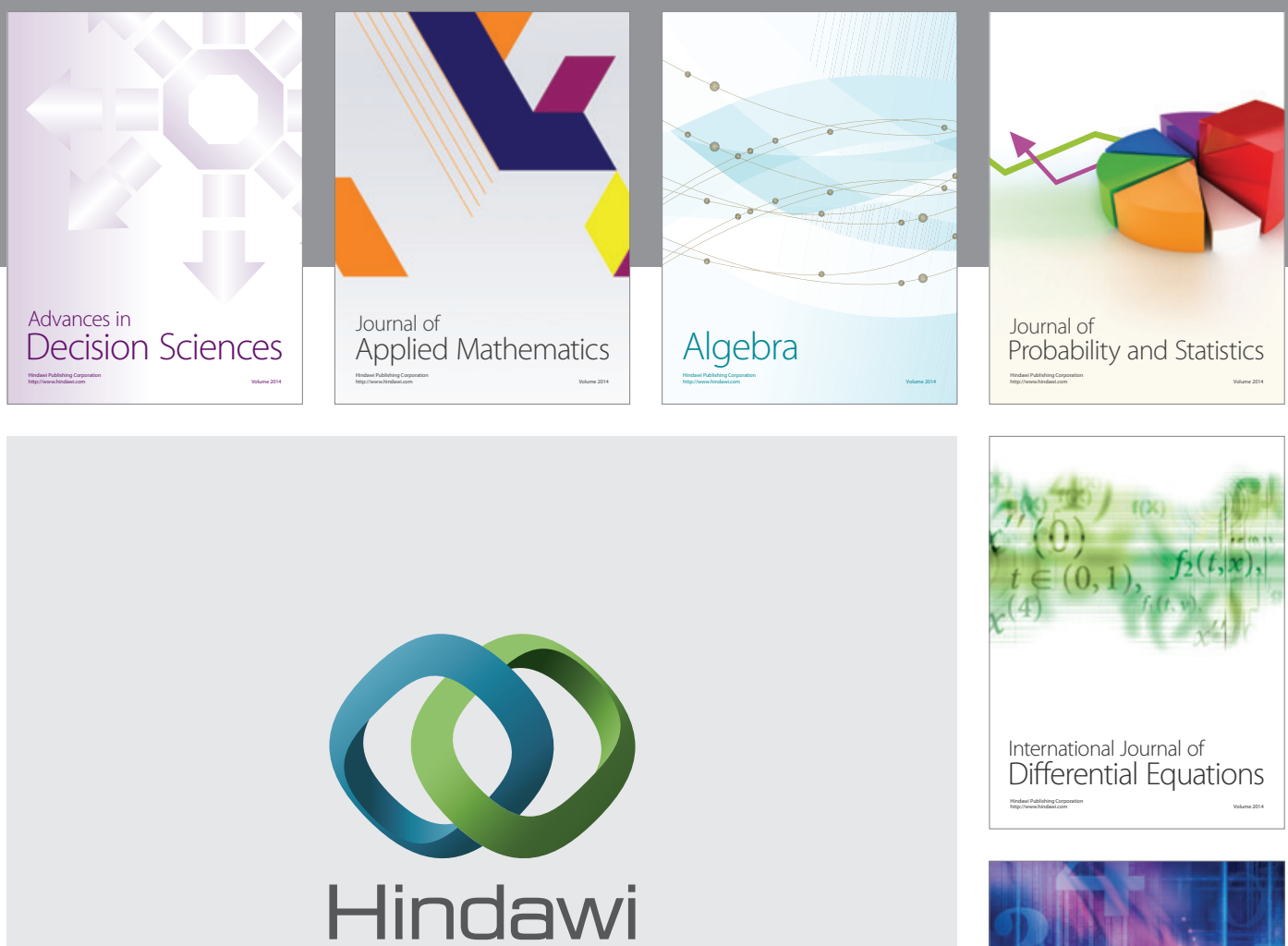

Submit your manuscripts at http://www.hindawi.com
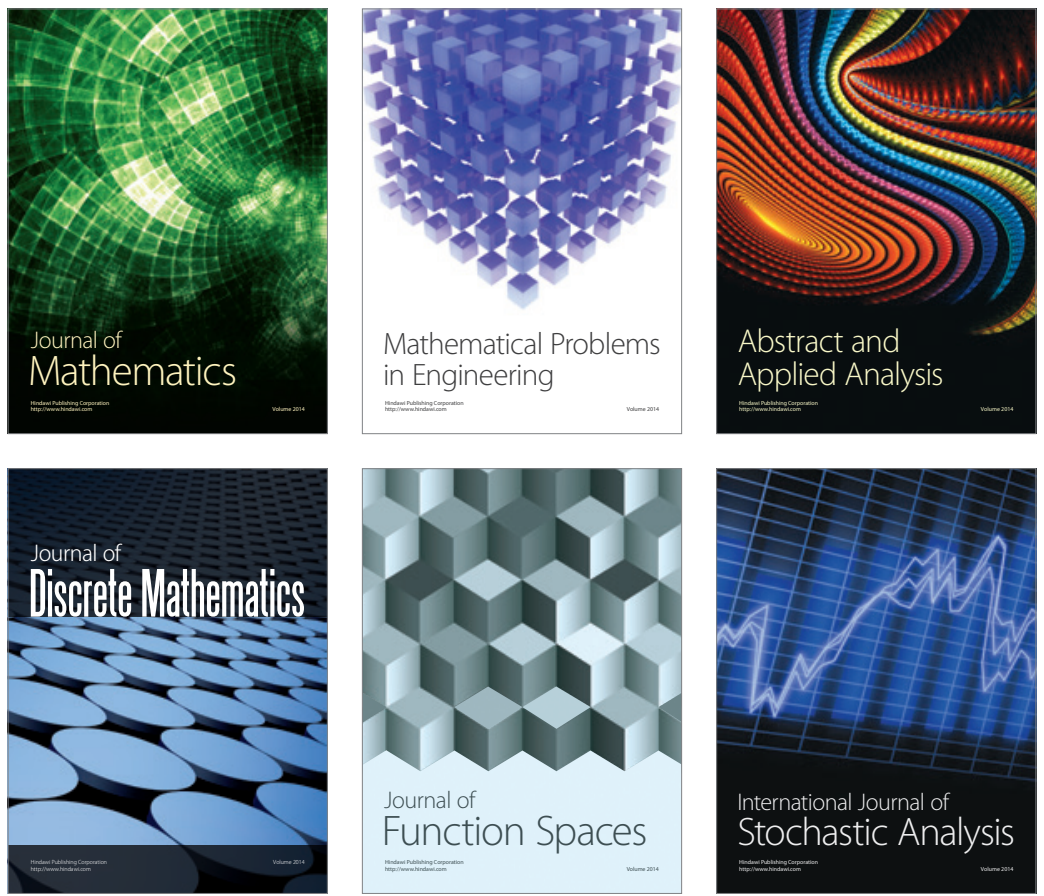

Journal of

Function Spaces

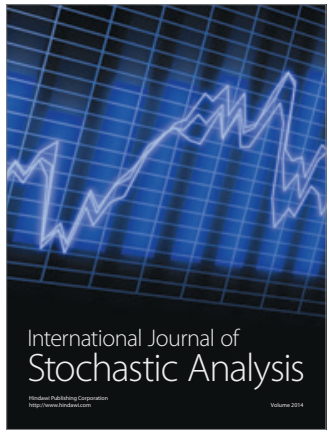

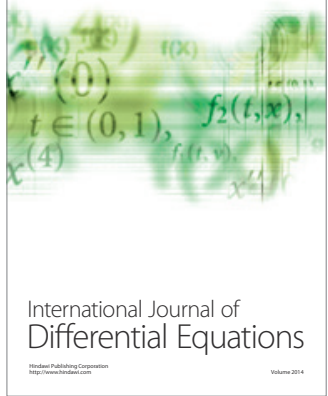
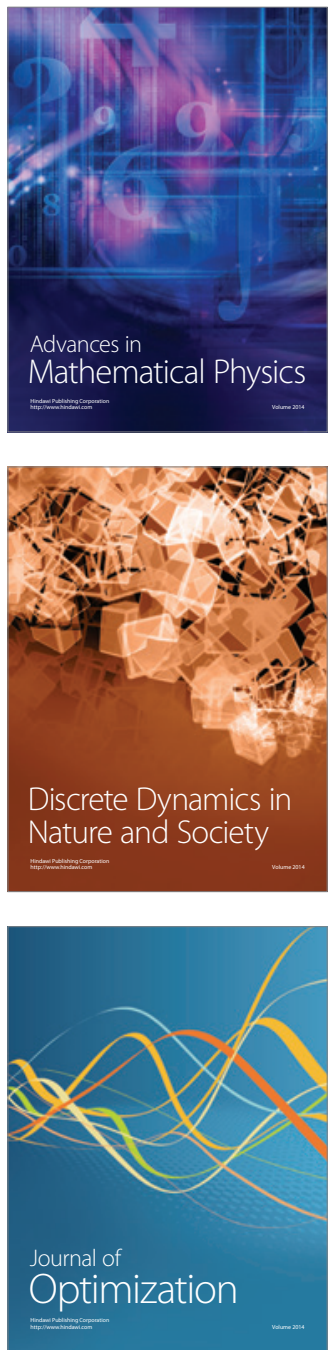